\title{
Epidemics and Dimensionality in Hierarchical Networks
}

\author{
Da-Fang Zheng ${ }^{1,2, *}$, P.M. Hui ${ }^{3}$, Steffen Trimper ${ }^{2}$, and Bo Zheng ${ }^{1,2}$ \\ 1 Zhejiang Institute of Modern Physics, Zhejiang University, \\ Hangzhou 310027, People's Republic of China \\ 2 Fachbereich Physik, Martin-Luther-Universität, D-06099 Halle, Germany and \\ ${ }^{3}$ Department of Physics, The Chinese University of Hong Kong, Shatin, Hong Kong, China
}

\begin{abstract}
Epidemiological processes are studied within a recently proposed hierarchical network model using the susceptible-infected-refractory dynamics of an epidemic. Within the network model, a population may be characterized by $H$ independent hierarchies or dimensions, each of which consists of groupings of individuals into layers of subgroups. Detailed numerical simulations reveal that for $H>1$, global spreading results regardless of the degree of homophily of the individuals forming a social circle. For $H=1$, a transition from global to local spread occurs as the population becomes decomposed into increasingly homophilous groups. Multiple dimensions in classifying individuals (nodes) thus make a society (computer network) highly susceptible to large scale outbreaks of infectious diseases (viruses).
\end{abstract}

PACS Nos.: 89.75.Hc, 87.23.Ge, 89.65.-s

Keywords: Epidemics; Hierarchical networks; Homophily

\footnotetext{
* Corresponding author.

E-mail address: dfzheng@zjuem.zju.edu.cn.
} 


\section{INTRODUCTION}

Epidemics of all kinds are costly. The influenza in the early 20th century and the outbreak of SARS in 2003 claimed precious lives, and the spreading of viruses on the internet has led to much economic loss when computer systems of governments and airlines were attacked. Recent breakdowns of major power networks in North America and in Europe also brought great loss and inconvenience to millions of people. A less damaging example is, perhaps, that of the spread of a rumor in a community. Epidemiological processes, together with percolation and problems on searchability, are the most interesting dynamical problems in a system of connected entities 1, 2, 3, 4]. There are two key factors in the study of epidemic spreading, namely how infection and recovery occur and how people or computers are connected. The latter is best described in terms of networks or graphs [2, 3, 4] in which the nodes representing people, communities, power plants, or computers are connected by links representing the acquaintance among people or the connections between computers. The most important question is the extent to which a disease (virus) may propagate. Since infections take place through direct contact between infected (I) and susceptible (S) nodes, the underlying structure of the network understandably plays a determining role on the spread of diseases [5].

The Susceptible-Infected-Refractory (or recovered/removal) (SIR) model [1, 6, 7] has been widely used in different forms for studying epidemiological processes such as the spread of influenza (see, for example, Ref. [8]) and SARS [9, 10], and also for the spread of rumors [11, 12]. In its simplest form, a node takes on one of three states, namely $\mathrm{S}, \mathrm{I}$ or $\mathrm{R}$. The R-state represents the case in which an infected node becomes recovered and immunized or, unfortunately dead. For computer networks, the R-state may represent recovery with the virus removed and anti-virus software installed. A node in the R-state is not infectious and will not become infected again. The underlying connectivity obviously plays a decisive role. For nodes connected randomly, a substantial proportion becomes infected and eventually recovered from an epidemic 13]. However, when a regular network is gradually re-wired into a small-world network [2, 3, 4, 14, 15] and then a random network, it was found that a threshold exists on the degree of disorderness below which the disease can only spread locally, but not globally [11, 12].

Networks have fascinating structural and dynamical properties, and they have wide ap- 
plications in physical, biological, social, economic, and computer systems [2, 3, 4, 16, 17]. Physicists have made important contributions to the understanding of networks in recent years. Structurally, the small-world networks [14, 15] and the growing scale-free networks 18] have been extensively studied [2, 3, 4]. Depending on the model, the degree distribution may show power law or exponential forms. As the underlying connectivity plays a decisive role in the studies of dynamical processes, a good network model that reflects the structural properties is therefore required for modelling epidemics or other dynamical problems in a connected population, or in systems in which the entities are grouped in a hierarchical fashion as in a population. The hierarchical model of searchable social networks proposed recently by Watts et al. [19] captures the essential ingredients of a network model of connected population and has enjoyed much attention. The model is physically transparent in that it is motivated by the observation that individuals within a population are grouped according to their function into different dimensions, for example, their occupation, hobby, home district, etc. The model exhibits the phenomena of "six degrees of separation", which was discovered by Travers and Milgram in the 1960's [20] and has been tested in a world-wide experiment on the internet [21].

Recent works on networks on robustness of networks under attacks [22] and ways to prevent attacks 23] indicate that, again, the connectivity of a network is important. It is, therefore, important to study the dynamical aspects of the successful social network model of Watts et al. [19]. In the present work, we present detailed results of numerical simulations on the extent of a spread of disease or virus in the hierarchical social network model of Watts et al. within the SIR epidemic dynamics. It is found that the spread may be local or global if the population can be partitioned in a unique way. However, when the partition of the population becomes multi-dimensional, the result is different from that of the searchability problem. It is found that the spread is always global.

The plan of the paper is as follows. In Sec.II, we motivate the hierarchical structure and describe how links are established between nodes in a hierarchical structure with a tunable degree of homophily. In Sec.III, we describe the implementation of the SIR dynamics and discuss the results on the spread of an epidemic in populations that can be classified into one and two hierarchies. Section IV summarizes our findings. 


\section{NETWORK MODEL OF CONNECTED POPULATION}

The model of Watts et al. was motivated by the general structure in the groupings of individuals in a society as shown in Fig.1 [19]. The highest level can be regarded as a population of $N$ individuals or nodes. These $N$ nodes may then be classified or partitioned into $b$ groups. Each group can further be divided into $b$ subgroups and so on. After $(l-$ 1) divisions the structure has a total of $l$ levels and ends at a level where an individual belongs to a close functional group of size $g$, where $g$ typically is of the order of $10^{1}$ to $10^{2}$. Individuals belonging to the same lowest-level subgroup have the highest chance of becoming most similar or getting acquaintance. For example, all physicists in academia can be classified roughly by their research area, say condensed matter physics or nuclear physics, and further classified by their subfields, e.g., magnetism or superconductivity, and then further grouped by their specific research interests, e.g., topics grouped into the same session in a conference or with the same code in the PACS classification scheme. Obviously the divisions are usually not unique. Physicists may be grouped geographically based on the region their institution is located, and then the country, state, county, department, and research groups. A population of individuals or a collection of nodes may thus be characterized by $H$ hierarchies or dimensions, each of which takes on the structure shown in Fig.1. The structure of the network of $N$ nodes is then characterized by the parameters $H, b, l$, and $\langle g\rangle$ with $N=\langle g\rangle b^{l-1}$, where $\langle g\rangle$ is the average size of the lowest-level functional subgroups.

An important quantity within a hierarchy is the social distance $x_{i j}$ that measures the similarity between two nodes $i$ and $j$. For nodes belonging to the same lowest-level subgroup in a given hierarchy, $x_{i j}=1$, otherwise $x_{i j}$ is the number of levels from the lowest for which the two nodes belong to the same group. Hence the largest separation is $l$ in a given hierarchy. For $H>1$, an important geometrical feature is that for nodes $i$ and $j$ with $x_{i j}=1$ in one hierarchy and $j$ and $k$ with $x_{j k}=1$ in another, $x_{i k}$ is in general not small. For example, it is unlikely that the colleague in the office next door knows your collaborator in another country. Within the context of epidemics in a community, the hierarchies may be regarded as family, relatives, friends, and so on; or neighbors in the same apartment building, same neighborhood, same district and so on. While the model was motivated by the structure in groupings within a society, it is obvious that many well-structured systems, e.g. computer 
clusters, are connected in a similar fashion.

Computationally, a social network with a tunable degree of homophily can be constructed as follows [15]. For a population of $N$ individuals with social structure as shown in Fig.1, the individuals are first assigned randomly into the lowest subgroups with an average size $\langle g\rangle$ in each of the $H$ hierarchies. Links between individuals are established as follows. For $H>1$, a hierarchy is randomly chosen. Two nodes $i$ and $j$ are then selected randomly within the chosen hierarchy. A link, specifying that $i$ and $j$ are friends and hence become acquaintance, is established with a probability $P\left(x_{i j}\right)=\exp \left(-\alpha x_{i j}\right) / \sum_{n=1}^{l} \exp (-\alpha n)$ depending on $x_{i j}$ of the chosen nodes in the chosen hierarchy. No duplicated links between $i$ and $j$ are allowed. Here, the parameter $\alpha$ is a measure of homophily of the system. The process is then repeated until a mean number of $\langle z\rangle=\langle g\rangle-1$ links are established for each node (individual) in the system. This guarantees that for $\alpha \gg-\ln b$ and $H=1$, only links between nodes with small separation are probable and the individuals are connected only to those most similar in character, leading to isolated subgroups of nodes. For $\alpha=-\ln b$, links between individuals with any social distance are equally probable and a random network results, with the notion of similarity between nodes loses its meaning. For intermediate values of $\alpha$, i.e., $\alpha \approx 1$, the network shows small-world features. Here, we aim at studying the extent of an epidemic as a function of $\alpha$ for systems with $H=1$ and $H=2$, respectively.

\section{EPIDEMIC MODELLING AND RESULTS}

We model epidemics on the social network model by the standard SIR dynamics, which is implemented as follows [6, 7]. Initially, all nodes are in the S-state and one node is randomly chosen for infection. At each timestep, a node $i$ is randomly chosen among all the infected (I) nodes. A neighbor (friend) $j$ is then selected randomly among all the neighbors of node $i$, i.e., those with a link connected to $i$. Note that the effects of dimensionality $H$ is built in through the construction of links. If node $j$ is susceptible, it becomes infected and the

chosen node $i$ remains in the I-state; otherwise (i.e., node $j$ is either I or R) the state of node $j$ remains unchanged and the chosen node $i$ becomes recovered $(\mathrm{R})$ at the end of the timestep. As time evolves, the number of R-nodes (S-nodes) increases (decreases); while the number of I-nodes increases initially and then eventually drops to zero. The number of R-nodes at the end of the epidemic is denoted by $N_{R}$. 
We performed extensive numerical simulations to explore the effects of the structural parameter $\alpha$ and the dimensionality or number of hierarchies $H$. For each set of parameters, data are collected over $10^{3}-10^{4}$ runs, each of which corresponds to a different realization of the network and a different initially infected node. We take $b=2,\langle g\rangle=10$ and hence $\langle z\rangle=\langle g\rangle-1=9$. Results for $H=1$ are shown in Fig.2. The structural effects on epidemics can be studied via the probability distribution function $P\left(N_{R}\right)$ for different values of $\alpha$ and different population sizes $N$. For $\alpha=10 \gg-\ln 2, P\left(N_{R}\right)$ is independent of $N$ and peaks at $N_{R} \approx 8$, with no network having $N_{R}>25$ (see Fig.2(a)). The independence on the population size $N$ implies that the spread is local, for cases of large $\alpha$. This is the case of "regular networks" consisting of isolated subgroups in which only local spread at the lowest level is possible. Each subgroup can be regarded as a small fully connected network. Previous studies showed that about $79 \%$ of nodes in a large fully-connected network eventually recovered [13]. Using this result as an estimation gives $0.79\langle g\rangle \approx 8$ for the size of a spread, in good agreement with the peak value observed. As only the mean $\langle g\rangle$ is fixed, each cluster has a different $g$ leading to a distribution $P\left(N_{R}\right)$ that extends to $N_{R}=25$. Simulations using different values of $\langle g\rangle$ reveal a corresponding shift in $N_{R}$ at which $P\left(N_{R}\right)$ shows a maximum. For the small-world regime corresponding to $\alpha \approx 1$ (see Fig.2(b)), $P\left(N_{R}\right)$ is again $N$-independent and decays exponentially with runs in which the spread can be up to $N_{R} \approx 10^{3}$. Geometrically, there exist some links of longer social distance as $\alpha$ decreases, leading to larger but still localized spreads. As $\alpha$ decreases towards the random network limit of $\alpha=-\ln 2, P\left(N_{R}\right)$ takes on the form of two disjoint parts (see Fig.2(c)): (i) one that decays exponentially near $N_{R} \approx 0$ (inset) characterizing the small fraction of runs with small-scale epidemics due to the existence of isolated clusters and (ii) a $\mathrm{N}$-dependent gaussian distribution characterizing global spreading centered at a value $\overline{N_{R}}$ that scales linearly with $N$. Writing $\overline{N_{R}}=a(\langle g\rangle) N$, we found $a \approx 0.66$ for $\langle g\rangle=10$. Extensive simulations reveal that $a(\langle g\rangle)$ approaches 0.79 , the value for fully-connected networks, as $\langle g\rangle$ increases. For $H=1$, the consequence of an epidemic depends sensitively on the structural parameter $\alpha$.

Social networks usually correspond to $H>1$. For $H>1$, the characteristic features of an epidemic is very different from that in $H=1$. The most striking result is that for $H \neq 1$, an epidemic can always spread globally regardless of the degree of homophily (i.e., value of $\alpha$ ) in the network. Figure 3 shows the results for $H=2$. For fixed $N$ and $\langle z\rangle$, $P\left(N_{R}\right)$ becomes independent of $\alpha$ and takes on a form identical to that in Fig.2(c). The 
majority of the weight is represented by the gaussian distribution peaked at $\overline{N_{R}} \approx a(\langle g\rangle) N$, with $a(\langle g\rangle=10) \approx 0.66$ and increases for larger $\langle g\rangle$. This result should be contrasted with that of searchability on the same social network [19], which occurs for $H>1$ and $\alpha>0$ with increasingly restricted constraints on $H$ and $\alpha$ as $N$ increases. For epidemics, $H>1$ alone is sufficient for a global spread of the disease and the result remains valid for different values of $N$. The independence on $\alpha$ can be understood by the way that the links are constructed. Although a larger $H$ implies fewer links per hierarchy, the chance of nodes $i$ and $j$ being similar with a shorter $x_{i j}$ in one of the hierarchies is higher and so is the chance that a link exists between nodes $i$ and $j$, even for large values of $\alpha$. In other words, nodes $i$ and $j$ unknown to each other in one hierarchy has a chance of becoming connected when additional dimension(s) is (are) added. Thus an increase in $H$ has the effect of promoting the spread of a disease in a population by permeating through different connected nodes in different hierarchies. This is consistent with daily experience that a flu may spread first from a parent to a kid (linked nodes in a family) and then between the kids in school (linked nodes in school) and so on. We have also studied the case of $H=3$ and obtained similar results.

To further illustrate the qualitative difference between $H=1$ and $H=2$ networks, we show the mean fraction of R-nodes, $r \equiv N^{-1} \sum_{N_{R}=0}^{N} N_{R} P\left(N_{R}\right)$, as a function of $\alpha$ in Fig.4. For $H=1$, the large $\alpha(\alpha \gg 1)$ regime gives $r \sim\langle g\rangle / N \approx 0$ corresponding to the case in Fig.2(a), while the small $\alpha(\alpha<\ln b)$ regime gives an $N$-independent value of $r \approx 0.66$ corresponding to the case in Fig.2(c). The fraction $r$ drops sharply in a small intermediate range of $\alpha(\ln 2<\alpha<2)$ corresponding to the small-world regime [19]. This feature is similar to that observed in the spreading of rumors in a small-world construction [11, 12]. In this regime, links between nodes with large dissimilarity exist and hence $N_{R} / N$ increases as $\alpha$ decreases. One would expect that the transition becomes sharper as $N$ increases, as $N_{R}$ is restricted only by the connections and is insensitive to $N$ in the intermediate regime. For $H=2$, the behavior is qualitatively different in that $r$ stays at a higher level for all values of $\alpha$ and the transition disappears as a result of the effect of the extra hierarchy for hooking up individuals in a population. 


\section{SUMMARY}

In summary, epidemiological processes are studied on a hierarchical social network within the SIR model. For population characterized by $H>1$ independent hierarchies, global spreading results regardless of the structural parameter $\alpha$ and hence the homophily of individuals forming a social circle. This result should be contrasted with that of searchability problems in that $H>1$ alone is sufficient for a global spread of a disease within the SIR dynamics. For $H=1$, a transition from global to local spread occurs as the population becomes decomposed into increasingly homophilous groups when the structural parameter $\alpha$ increases. Since social networks usually correspond to $H>1$, it is therefore very difficult to confine an epidemic, unless the "dimensionality" of the infected nodes can be reduced at least temporarily. One way to achieve this is to quarantine the infected nodes, which is known to be an effective way in handling infectious diseases. It will also be interesting to extend the present study to other models of epidemics such as the SIS [5] and SIRS [24] models.

\section{Acknowledgments}

This work was partly supported by the National Natural Science Foundation of China under Grant No. 70471081 and No. 70371069, by a DFG grant under Grant No. TR 3000/33, and by a grant from the Research Grants Council of Hong Kong SAR Government under Grant No. CUHK4241/01P. 
[1] O. Diekmann, J.A.P. Heesterbeek, Mathematical Epidemiology of Infectious Diseases: Model Building, Analysis and Interpretation, John Wiley \& Sons, New York, 2000.

[2] R. Albert, A.-L. Barabási, Rev. Mod. Phys. 74(2002)47.

[3] S.N. Dorogovtsev, J.F.F Mendes, Adv. Phys. 51(2002)1079.

[4] S.N. Dorogovtsev, J.F.F Mendes, Evolution of Networks, Oxford University Press, New York, 2003.

[5] R. Pastor-Satorras, A. Vespignani, Phys. Rev. Lett. 86(2001)3200.

[6] R.M. Anderson, R.M. May, Infectious Diseases of Humans: Dynamics and Control, Oxford University Press, Oxford, 1991.

[7] J.D. Murray, Mathematical Biology, Springer, Berlin, 1989.

[8] Z. Liu, Y.C. Lai, N. Ye, Phys. Rev. E 67(2003)031911.

[9] S. Riley et al., Science 300(2003)1961.

[10] M. Lipsitch et al., Science 300(2003)1966.

[11] D.H. Zanette, Phys. Rev. E 64(2001)050901(R).

[12] D.H. Zanette, Phys. Rev. E 65(2002)041908.

[13] A. Sudbury, J. Appl. Probab. 22(1985)443.

[14] D.J. Watts, S.H. Strogatz, Nature (London) 393(1998)440.

[15] D.J. Watts, Small Worlds: The dynamics of networks between order and randomness, Princeton, New Jersey, 1999.

[16] S.H. Strogatz, Nature 410(2001)268.

[17] M.E.J. Newman, Comput. Phys. Commun. 147(2002)40.

[18] A.-L. Barabási, R. Albert, Science 286(1999)509.

[19] D.J. Watts, P.S. Dodds, M.E.J. Newman, Science 296(2002)1302.

[20] J. Travers, S. Milgram, Socimetry 32(1969)425.

[21] P.S. Dodds, R. Muhamad, D.J. Watts, Science 301(2003)827.

[22] B. Shargel, H. Sayama, I.R. Epstein, Y. Bar-Yam, Phys. Rev. Lett. 90(2003)068701.

[23] R. Cohen, S. Havlin, D. ben-Avraham, Phys. Rev. Lett. 91(2003)247901.

[24] M. Kuperman, G. Abramson, Phys. Rev. Lett. 86(2001)2909. 


\section{FIGURE CAPTIONS}

Figure 1: Schematic diagram of the groupings of individuals in a hierarchical social network. A population of $N$ nodes are classified into $b$ groups. Each group is further divided into $b$ subgroups and so on. After $(l-1)$ divisions, the lowest-level subgroups consist of $g$ individuals. There $x_{i j}$ is the social distance between nodes $i$ and $j$. A population can, in general, be characterized by $H$ such hierarchies.

Figure 2: The probability distribution function $P\left(N_{R}\right)$ of the number of R-nodes at the end of an epidemic in a population characterized by $H=1$ hierarchy. The structural parameter takes on (a) $\alpha=10$, (b) $\alpha=1$, (c) $\alpha=-\ln 2$. Different population sizes $N=1280$ (circles), 2560 (squares), and 5120 (triangles) corresponding to $l=8,9,10$ are studied. Each data point represents an average over $10^{4}$ runs. The inset in (c) shows the portion of $P\left(N_{R}\right)$ for small $N_{R}$.

Figure 3: The probability distribution function $P\left(N_{R}\right)$ in a population of $N=2560(l=9)$ characterized by $H=2$ hierarchies. The structural parameter takes on $\alpha=10$ (circles), $\alpha=1$ (squares), and $\alpha=-\ln 2$ (triangles). Each data point represents an average over $10^{4}$ runs. $P\left(N_{R}\right)$ signifies a global spread of disease regardless of the structural parameter for $H>1$.

Figure 4: The fraction of R-nodes at the end of an epidemic as a function of the structural parameter $\alpha$ in a population of $N=1280$ (circles), 2560 (squares), and 5120 (triangles) characterized by (a) $H=1$ and (b) $H=2$ hierarchies. Each data point represents an average over $10^{3}$ runs. For $H>1$, global spreading occurs for all values of $\alpha$, while a transition from global to local spreading occurs for $H=1$. 
Zheng et al

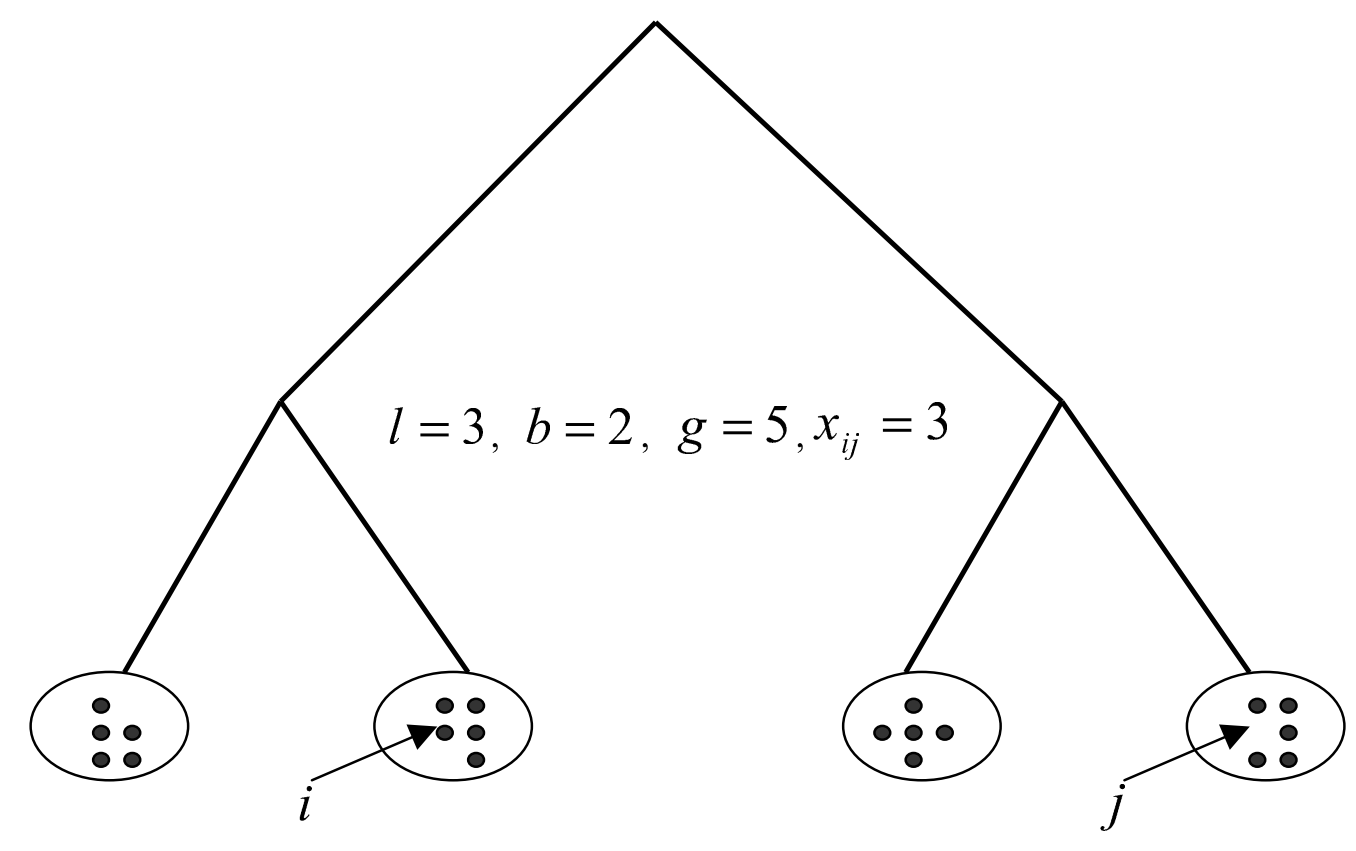

Figure 1 



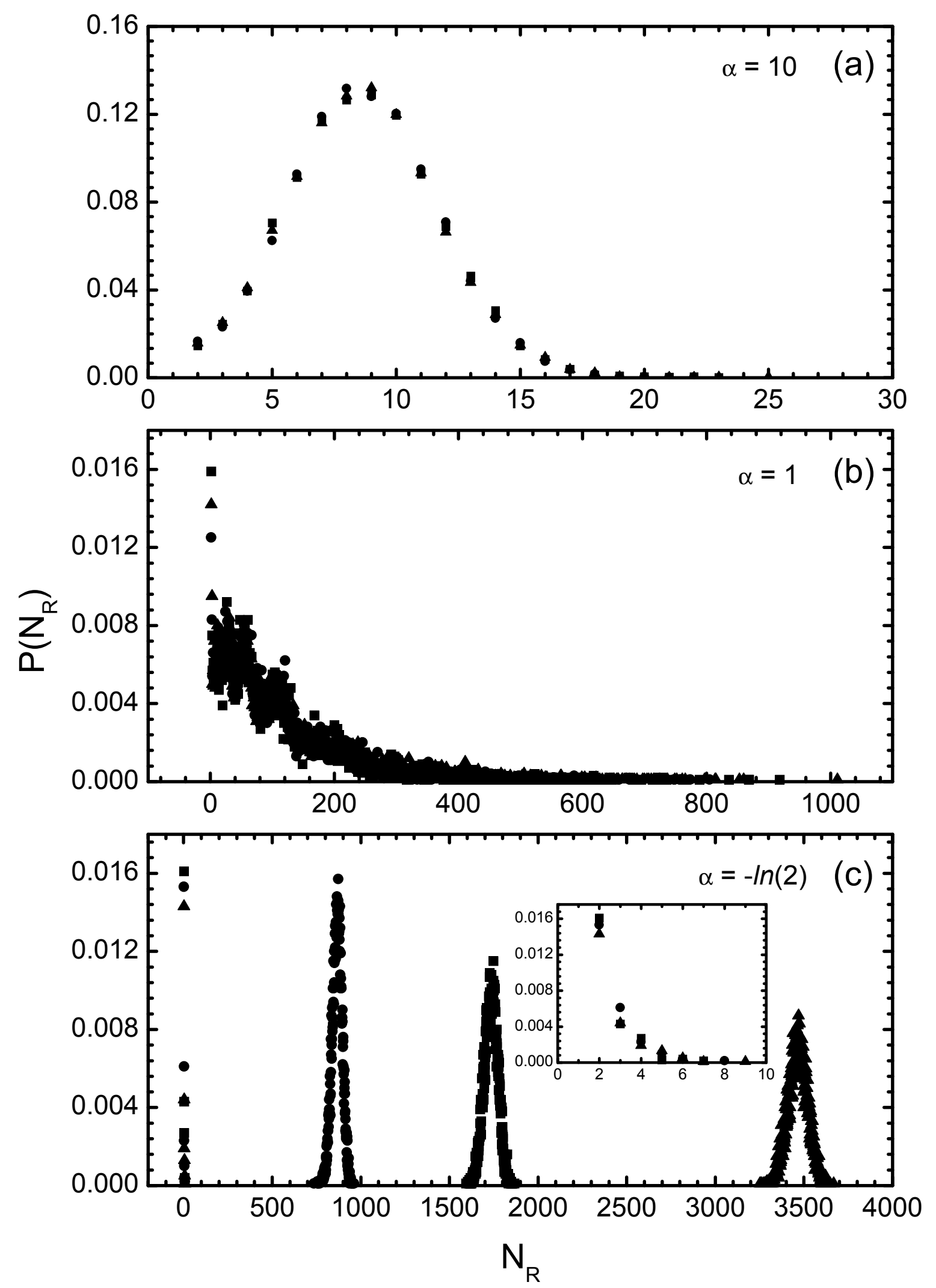

Figure 2 
Zheng et al

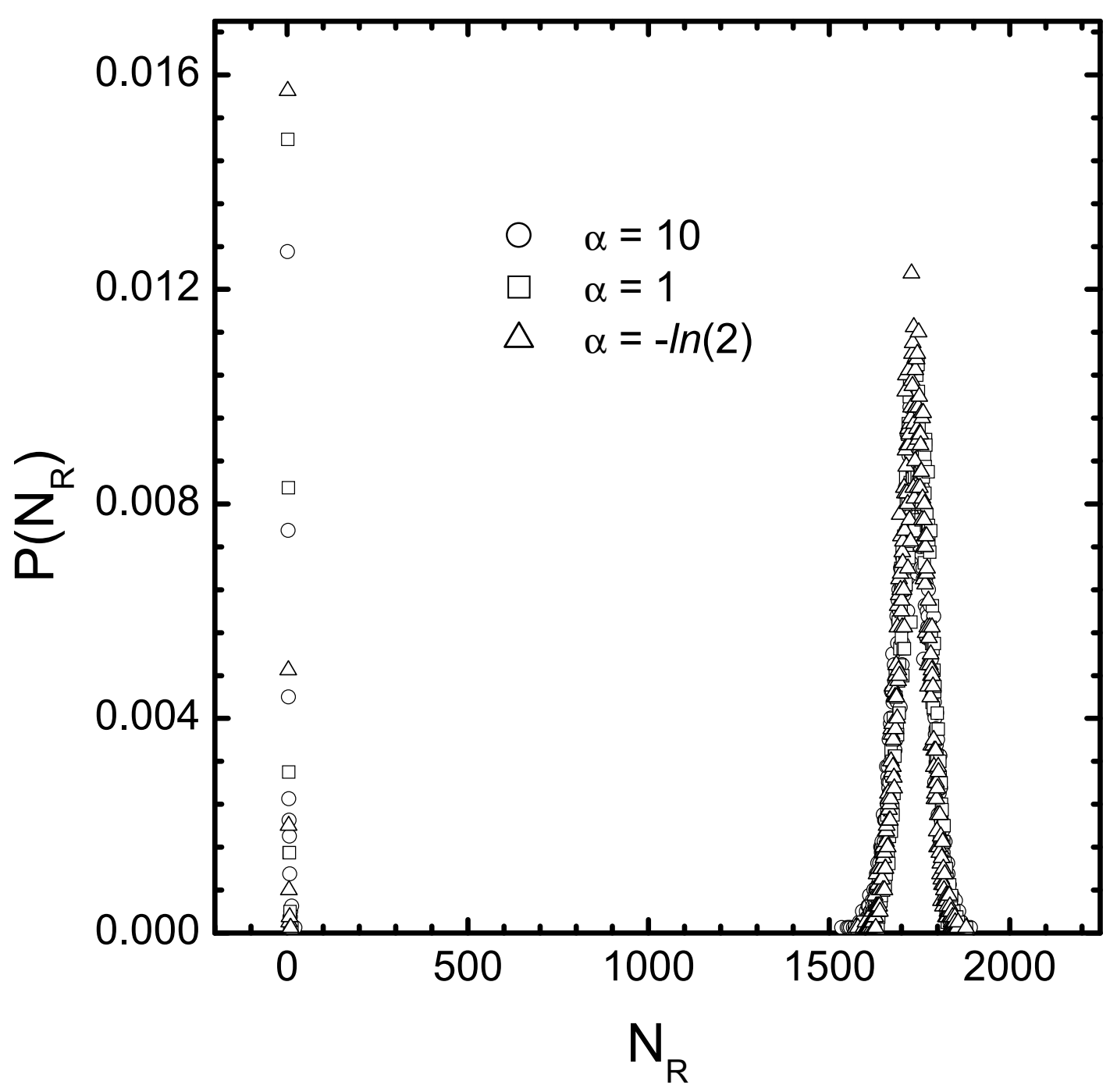

Figure 3 
Zheng et al
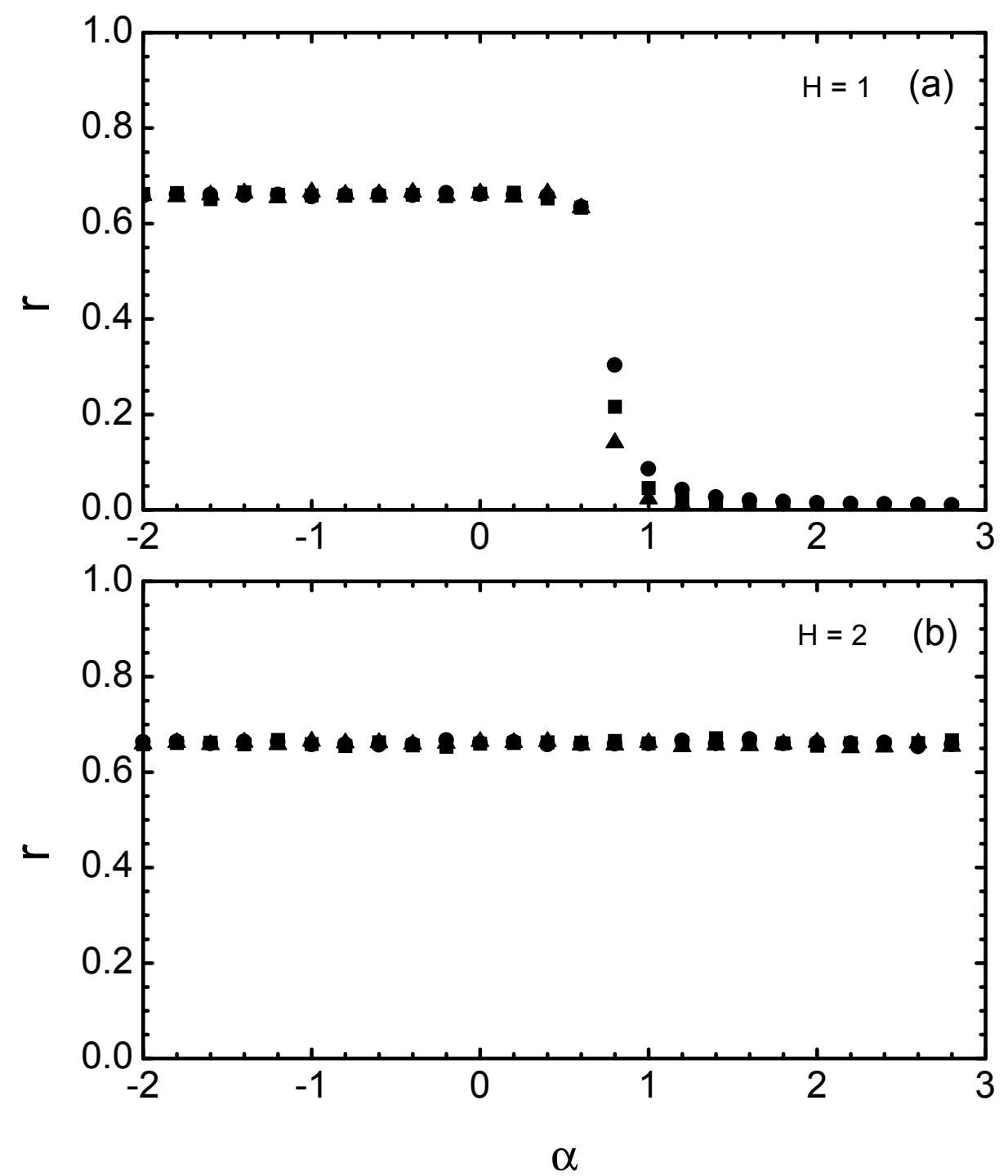

Figure 4 